\title{
RELATORÍAS DEL CONGRESO INTERNACIONAL DE EXCLUSIONES PROBATORIAS EN EL ENTORNO DE LA INVESTIGACIÓN Y PRUEBA ELECTRÓNICA
}

\author{
PIEDAD GONZÁLEZ GRANDA \\ Catedrática de Derecho Procesal \\ Universidad de León
}

En la Facultad de Derecho de la Universidad de León tuvo lugar los pasados días 3 y 4 de octubre -y con un muy alto nivel de participación- la celebración del Congreso Internacional de Derecho Procesal Exclusiones probatorias en el entorno de la investigación y prueba electrónica. Evento que a lo largo de dos jornadas distribuidas en diversas sesiones cada una de ellas y bajo la dirección de la Profesora Dra. Piedad González Granda (Catedrática de Derecho Procesal de la Universidad de León), reunió a profesores de diversas Universidades españolas y de otros países, así como a determinados expertos de otros ámbitos del mundo jurídico, con el afán de propiciar y difundir la disertación conjunta en torno al tema general que da título al Congreso, desde enfoques y perspectivas diversas.

La organización del Congreso trajo causa del patrocinio de la Fundación Privada Manuel Serra Domínguez -cuyo objetivo principal es precisamente la promoción y el fomento de la investigación del Derecho Procesal- a través de la concesión de un Proyecto en promoción de la excelencia el pasado año 2018, mediante la correspondiente convocatoria pública. Asimismo, figura como patrocinadora la Editorial REUS, con el compromiso de publicación del resultado de las Ponencias y Comunicaciones seleccionadas. También ha de mencionarse al Vicerrectorado de Relaciones Institucionales y con la Sociedad, que a través de la convocatoria pública de ayudas para la organización de Congresos, ha contribuido a su realización; del mismo modo ha de mencionarse al Consejo Social de esta Universidad, que en atención principalmente al carácter internacional del Congreso, ha querido asimismo colaborar para hacer posible su realización.

Estructurado el Congreso en tres sesiones, comenzó la primera de ellas en la mañana del día 3 de octubre con el Acto de Inauguración y Presentación del Congreso, que contó con la asistencia del Rector de la Universidad de la León y con el Director de la Fundación Privada de Derecho Procesal Manuel Serra Domínguez. Acto seguido, dio comienzo la exposición de las Ponencias a cargo de los profesores invitados de las 
Universidades extranjeras que dieron rango internacional al Congreso. La primera de ellas, con el título Diligencias intrusivas y Prueba ilícita, con especial referencia a las Fuentes de Prueba digital en el ordenamiento chileno, corrió a cargo de D. Raúl Núñez, Profesor de Derecho Procesal de la Pontificia Universidad Católica de Valparaíso (Chile). Y la segunda, bajo el título La exclusión de la Prueba en el Proceso Canónico y su proyección en el Proceso civil italiano, fue expuesta por D. Andrea Bettetini, Catedrático de Derecho Canónico y Eclesiástico de la Universidad Católica de Milán.

La segunda sesión tuvo lugar el mismo día 3 de octubre por la tarde, con una Mesa Redonda en torno al tema de Las exclusiones probatorias en el entorno de la investigación electrónica del Proceso Penal, que contó con la presencia de tres Ponentes: D. José Francisco Etxeberría Guridi, Catedrático de Derecho Procesal de la Universidad del País Vasco, que centró su exposición en la obtención de perfiles de ADN mediante la Orden Europea de investigación: las dos caras de la misma moneda; Dá Elvira Tejada de la Fuente, Fiscal de Sala del TS y Coordinadora de Criminalidad Informática de la Fiscalía General del Estado, que disertó sobre La incorporación al proceso de los datos almacenados (artículos 588.ter.j-588.ter.m LECrim.); y D. Pedro Álvarez Sánchez de Movellán, Profesor Titular de Derecho Procesal de la Universidad de León, que realizó su exposición sobre Ponderaciones judiciales en materia de prueba prohibida y garantías para la nueva investigación en el proceso penal.

La tercera sesión tuvo lugar en la mañana del día 4 de octubre, con una Mesa Redonda en torno a Las exclusiones probatorias en el entorno de la Prueba Digital en el orden jurisdiccional civil, que contó asimismo con la intervención de tres Ponentes: D. Valentín Cortés Domínguez, Catedrático de Derecho Procesal de la Universidad Autónoma de Madrid y abogado, con un discurso centrado en Prueba prohibida en el Proceso Civil: particular vulnerabilidad de los derechos fundamentales en el entorno electrónico; Dạ María Jesús Ariza Colmenarejo, Profesora Titular de Derecho Procesal de la Universidad Autónoma de Madrid, con el título Tratamiento de los datos contenidos en la Prueba digital incorporada al proceso; y Da Piedad González Granda, Catedrática de Derecho Procesal de la Universidad de León y directora del evento, con una exposición centrada en los Problemas específicos del acceso a las Fuentes de Prueba Digital en el orden jurisdiccional civil.

Seguidamente tuvo lugar la presentación y exposición de las siguientes Comunicaciones seleccionadas: La exposición de los hijos menores en redes sociales como prueba para la privación de la patria potestad (Da Cristina Llamas Bao, Profesora Asociada de la Universidad de León y abogada del ICAL); La identificación fenotípica del autor del delito y su valoración en el proceso penal (Da Susana Álvarez de Neyra Kappler, Profesora Contratada Doctora de Derecho Procesal de la Universidad Autónoma de Madrid; Exclusiones probatorias a instancia de la persona jurídica (D. Felipe Pérez del Valle, abogado ejerciente del ICAL); Proceso penal y volatilidad/mutabilidad de las fuentes de prueba electrónicas: sobre la conveniencia y el modo de asegurarlas eficazmente ( $D$ a Eva Isabel Sanjurjo Ríos, Profesora 
Contratada Doctora de Derecho Procesal de la Universidad de León); y La cadena de custodia en la prueba digital: España vs EEUU (Da Margarita Simarro Pedreira, investigadora del Programa de Doctorado "Responsabilidad Jurídica multidisciplinar" de la Universidad de León y abogada del ICAL). Excusaron su asistencia las autoras de las siguientes Comunicaciones seleccionadas: Dạ Pilar Martín Ríos, Profesora Titular de Derecho Procesal de la Universidad de Sevilla (El valor probatorio de información obtenido de un ordenador de uso compartido: a propósito de la STS 287/2017); y Dạ María Elena Laro-González, investigadora contratadas Predoctoral del Departamento de Derecho Procesal de la Universidad de Sevilla (Principio de proporcionalidad en la obtención, cesión y tratamiento de datos personales en materia penal).

El contenido de las Ponencias y Comunicaciones seleccionadas expuestas en el marco del Congreso se resume a continuación a través de las breves aportaciones realizadas por los propios autores. (Coordina el conjunto de las relatorías expuestas por orden alfabético Da María Santisteban Castro, Estudiante del Grado en Derecho de la Universidad de León, con acreditación de actividades investigadoras en el Área de Derecho Procesal en el período julio-septiembre del presente año 2019).

Ponencia: PONDERACIONES JUDICIALES EN MATERIA DE PRUEBA PROHIBIDA Y GARANTÍAS PARA LA NUEVA INVESTIGACIÓN EN EL PROCESO PENAL

Ponente: D. Pedro Álvarez Sánchez de Movellán, Profesor Titular de Derecho Procesal de la Universidad de León

Comienza la ponencia haciendo una referencia al status quaestiones de la prueba prohibida en el proceso penal. Se parte de la redacción del artículo 11. $1 \mathrm{LOPJ}$, que declara la ineficacia probatoria de pruebas obtenidas directa o indirectamente violentando los derechos o libertades fundamentales. Esta norma legal, guarda relación con la primera jurisprudencia del Tribunal Constitucional, tal y como se puede apreciar en la STS 114/1984, de 29 de noviembre. Esa doctrina jurisprudencial fundamentaba la ineficacia de la prueba ilícita como exigencia del derecho al proceso con todas las garantías y como exigencia de la debida protección de los derechos y libertades recogidos en el Título I, Capítulo II, Sección $1^{\text {a }}$ de la Constitución Española.

Con el paso del tiempo la doctrina se fue «moderando» y así lo vemos en la STC $81 / 1998$, de 2 de abril y en otras resoluciones del TC y del TS, a través de excepciones que limitan la virtualidad del referido art. 11. 1 LOPJ. Así podríamos hablar de una modulación de aquella norma, en base a excepciones de lo que se entiende que debe ser la regla general; que no es otra cosa que la disposición legal. Y así, se barajan categorías como la excepción de prueba independiente, la excepción del hallazgo casual, la excepción de confesión voluntaria o la excepción de buena fe, $\mathrm{y}$-de una forma particulamente intensa- la «excepción de la conexión de antijuridicidad». Por otra parte, la STC 97/2019, de 16 de julio, da lugar a un decisivo giro en esta materia en el sentido de desvincular la exclusión probatoria de la vulneración de derechos 
fundamentales, y esto, razonando la necesidad de acudir a la ponderación de aquella vulneración con el derecho (incierto y no recogido como tal en los textos normativos) a un «proceso justo y equitativo».

Esta incertidumbre da lugar a que, a la hora de aplicar el art. 11. 1 LOPJ, pensemos que se genera una inmensa inseguridad jurídica si se analizan las posibilidades de investigación a través de medios tecnológicos regulados en la Ley Orgánica 13/2015, de 5 de octubre, de modificación de la Ley de Enjuiciamiento Criminal para el fortalecimiento de las garantías procesales y la regulación de las medidas de investigación tecnológica. En esta regulación, las garantías de legalidad en las diligencias que allí se prevén,podríamos decir que se basan en tres pilares: por un lado, en la virtualidad de los principios rectores acuñados por años de jurisprudencia que, además de inspirar el régimen legal, son de directa aplicación; en segundo lugar, en el régimen de la resolución judicial que adopta tales medidas, donde la regulación del auto goza de una minuciosidad mayor que la regulación de la sentencia; y en tercer lugar, el régimen de la incorporación al proceso, de los datos almacenados, donde se dispone la forma en que los datos electrónicos conservados por los prestadores de servisios o personas que faciliten la comunicación, pueden ser cedidos para su incorporación al proceso con autorización judicial.

Vista la doctrina del TC y esta regulación de las medidas limitativas de los derechos del art. $18 \mathrm{CE}$, la cuestión es: ¿hasta qué punto las irregularidades cometidas en aquellas medidas de investigación tecnológica podrán ser definitivas para apreciar una exclusión probatoria? Pues bien, atendiendo a la potencialidad de estas medidas de investigación y a la enorme inmisión que tienen en el derecho a la intimidad, surge la duda de que se pueda estar poniendo el proceso penal al servicio de los posibles intereses de un Estado totalitario.

Ponencia: TRATAMIENTO DE LOS DATOS CONTENIDOS EN LA PRUEBA DIGITAL INCORPORADA AL PROCESO

Ponente: $\mathbf{D}^{\mathrm{a}}$. María Jesús Ariza Colmenarejo, Profesora Titular de Derecho Procesal de la Universidad Autónoma de Madrid

La protección de datos personales, una vez que se incorporan al proceso civil es, sin duda, uno de los temas más inquietantes en la actualidad. No es ajeno el hecho de que al proceso acceden multitud de datos, tanto de carácter personal, como de carácter no personal, y todo ese material se incorpora al Expediente Judicial Electrónico, cuya custodia corresponde al Letrado de la Administración de Justicia.

En relación con este tema, debemos atender a dos cuestiones fundamentales:

- Por un lado, a la delimitación del marco legislativo, compuesto por el Reglamento 2016/679, de 27 de abril (RGPD) del Parlamento Europeo, relativo a la protección de personas en lo que respecta al tratamiento de datos personales y a la libre circulación de estos datos, por la Ley Orgánica 3/2018, de 5 de diciembre, de 
Protección de Datos Personales y garantía de los derechos digitales, y la Ley Orgánica 7/2015, de 21 de julio, por la que se modifica la Ley Orgánica 6/1985, de 1 de julio, del Poder Judicial. a través de la cual, se incorpora el Capítulo I bis titulado «Protección de datos de carácter personal en el ámbito de la Administración de Justicia».

- Por otro lado, es preciso analizar las consecuencias de la digitalización de la Administración de Justicia, a través del Expediente Judicial Electrónico, y su repercusión en el tratamiento de datos personales e, incluso, los no personales. A tal efecto, existen múltiples repercusiones del derecho de autodeterminación informativa sobre los derechos de carácter procesal, lo cual requiere de una ponderación de intereses para que la tutela judicial no se vea mermada.

En ambos casos, las conclusiones están en la línea de considerar insuficiente la regulación, ya que, en ocasiones, se generan vacíos legales -en especial, en lo que a los responsables de datos se refiere-, y se produce una inadecuación de las disposiciones sobre protección de datos en relación con el principio de publicidad procesal y el derecho de las partes procesales de acceso al expediente. Problemas como la debida confidencialidad o la declaración de secreto de las actuaciones pueden llegar a poner en riesgo el derecho de defensa.

Ponencia: LA EXCLUSIÓN DE LA PRUEBA EN EL PROCESO CANÓNICO Y SU PROYECCIÓN EN EL PROCESO CIVIL ITALIANO

Ponente: D. Andrea Bettetini, Catedrático de Derecho Canónico y Eclesiástico de la Universidad Católica de Milán

La prueba constituye el eje del sistema procesal canónico, siendo un elemento que forma parte de la base del juicio, lo cual, no difiere del derecho secular.

Las partes pueden aportar probationes generis cuiuslibet (Canon 1527, §1 del Código de Derecho Canónico), o lo que es lo mismo, pruebas de cualquier naturaleza aunque, necesariamente, útiles y legítimas. Este principio, es de naturaleza atípica en relación a los medios de prueba, y aquí el Juez tiene el control tanto de la utilidad y de la relevancia de la prueba para el juicio de hecho -es decir, del factum probandum-, como el control respecto del cumplimiento de los requisitos y de las condiciones a las que el propio ordenamiento jurídico subordina la prueba para su admisión.

Por otro lado, el derecho a presentar todo medio de prueba para producir convicción, tiene, al menos, dos tipos de limitaciones, derivadas del objeto de la actividad probatoria y del respeto a ciertas formas y garantías. Entre estos, se mencionan, aquellos requisitos relativos a la pertinencia, a la relevancia o a la necesidad de la prueba, que algunos autores identifican como límites intrínsecos a la actividad probatoria, y aquellos requisitos vinculados a la licitud de la prueba, límites extrínsecos. 
La consecuencia de obtener una prueba ilícitamente, conlleva a que ésta no tenga ningún efecto $\mathrm{y}$, por tanto, su salida del proceso $\mathrm{y}$, la imposibilidad de tenerse en cuenta y deser valorada. A modo de ejemplo, no podrán ser valoradas las pruebas obtenidas en una entrada y registro que no cuente con la necesaria autorización judicial o consentimiento del titular, como tampoco se tendrá en cuenta una pericia que no acepte los principios antropológicos de la Iglesia Católica.

Por otra parte, si el Juez no admite por decreto, emitido de conformidad con el Canon $1590, \S 2$, una prueba, bien porque no la considera relevante o pertinente en ese supuesto, o bien porque la propia ley la excluye, se le otorga a la parte que ha presentado la prueba rechazada, el derecho de apelación, que se ejerce ante el mismo Juez que ha rechazado ese medio de prueba. Tal cuestión incidental, se debe concluir expeditissime, en el menor tiempo posible para evitar que las partes acudan al ejercicio de este medio con la finalidad de ocasionar dilaciones.

La exclusión de la prueba en el proceso eclesiástico tiene una mera relevancia canónica, tal y como afirmó la Casación italiana -secciones conjuntas civiles- en la resolución 14839/2011, de 6 de julio, donde se establecía que la actividad ejercida por el Juez eclesiástico en el proceso canónico -en el cual, como es lógico, el Juez se ajustará a lo establecido en las reglas procesales canónicas-, no podrán ser objeto de una evaluación o control por el Juez del Estado mientras esa actividad se encuentre dentro del propio marco del proceso canónico. Ello se justifica en virtud de la reserva exclusiva de la jurisdicción eclesiástica sobre la violación de las leyes eclesiásticas expresadas por el Canon 1401 del Código de Derecho Canónico, como en virtud a la regla de la separación e independencia de los órdenes, expresada por el art. 7 de la Constitución Italiana, una separación e independencia que constituyen la esencia del principio de la laicidad del Estado.

Sólo hay dos supuestos en los que la actuación del Juez de la Iglesia puede tener relevancia en el ordenamiento estatal:

- Cuando tal actuación pueda constituir delito.

- Cuando, a través de tal actuación, se vulneren los derechos fundamentales de las personas.

Ponencia: LA OBTENCIÓN DE PERFILES DE ADN MEDIANTE LA ORDEN EUROPEA DE INVESTIGACIÓN: LAS DOS CARAS DE UNA MISMA MONEDA

Ponente: D. José Francisco Etxeberria Guridi, Catedrático de Derecho Procesal de la Universidad del País Vasco

La Directiva 2014/41/CE del Parlamento Europeo y del Consejo, de 3 de abril de 2014, relativa a la Orden Europea de Investigación (OEI) en materia penal y su transposición al ordenamiento español mediante la Ley 3/2018, de 11 de junio, por la que se modifica la Ley 23/2014, de 20 de noviembre, de reconocimiento mutuo de resoluciones penales en la Unión Europea, para regular la Orden Europea de 
Investigación, constituyen un hito en el proceso de consolidación del Espacio Europeo de Justicia, Libertad y Seguridad. Mediante estos instrumentos se aborda la trascendental cuestión relativa a la cooperación judicial penal en el ámbito específico de la obtención de pruebas en la delincuencia con dimensión transfronteriza. Hasta el presente, la cooperación judicial penal en el seno de la UE se ha basado en el principio de reconocimiento mutuo de resoluciones judiciales. Sin embargo, la Directiva 2014/41 y la Ley 3/2018 de transposición, incorporan un novedoso mecanismo de cooperación en el que conviven el mencionado principio de reconocimiento mutuo junto con el tradicional mecanismo de asistencia judicial entre Estados.

Esta dualidad tiene una razón de ser. En materia de obtención de pruebas adquiere un particular protagonismo el debido respeto a los derechos fundamentales del ciudadano, pues con frecuencia, algunas medidas de investigación inciden en derechos del individuo de esa naturaleza. Esquemáticamente puede resumirse el diseño creado por la OEI de la siguiente manera: a) las medidas de investigación restrictivas de derechos fundamentales están sujetas a un doble control de legalidad y de garantías, por un lado, las propias del Estado de emisión, y, por otro lado, también las propias del Estado de ejecución, pues la normativa sobre la OEI posibilita que en este último se someta la OEI también a un control de legalidad, ampliando de forma considerable los motivos de denegación de la ejecución de la orden; b) las medidas de investigación no restrictivas de derechos fundamentales se ajustan en su emisión y ejecución al ya consolidado principio de reconocimiento, siendo más limitadas las facultades de control y de denegación por parte del Estado de ejecución.

Este doble mecanismo que consagra la OEI es de aplicación paradójicamente a la obtención de perfiles de ADN. Todo depende de si el perfil genético que interesa se encuentra ya incorporado a una base de datos en el Estado de ejecución o no. En el primer supuesto se considera el perfil de ADN como información obrante en una base de datos y se aplica a la OEI el principio de reconocimiento mutuo. En el segundo caso, hay que proceder a solicitar la ejecución de una intervención corporal para obtener muestras biológicas y realizar el examen genético correspondiente sobre las mismas. En este caso se incide de forma directa en los derechos fundamentales del individuo afectado y se activan las garantías correspondientes, no sólo en el Estado de emisión, también en el de ejecución.

Ponencia: PROBLEMAS ESPECÍFICOS DEL ACCESO A LAS FUENTES DE PRUEBA DIGITAL EN EL ORDEN JURISDICCIONAL CIVIL

Ponente: Da. Piedad González Granda, Catedrática de Derecho Procesal de la Universidad de León

En el marco de la Mesa Redonda "Las exclusiones probatorias en el entorno de la prueba electrónica en el orden jurisdiccional civil", y bajo el título de Problemas específicos del acceso a las fuentes de prueba digital en el orden jurisdiccional civil, analiza la autora el objeto de su Ponencia desde una perspectiva que gira en torno a los siguientes tres parámetros. En primer lugar, el entorno de la prueba digital, como 
especialidad -en sentido amplio- de la prueba. En segundo lugar, el alcance de las exclusiones probatorias - es decir, de los efectos de la llamada Prueba prohibida- fuera del proceso penal, y en concreto en el proceso civil. Y en tercer lugar, y en estrecha relación con lo anterior, el ámbito de los poderes del Juez y de las partes en los actos probatorios, siempre en constante búsqueda de equilibrio, con algunos cuestionamientos concretos y específicos en el entorno digital.

Con ese escenario de fondo -apasionante y bien trabado, en la medida en que dichos tres parámetros se caracterizan por su permanente construcción y revisión, dado el estado actual de la ciencia del Derecho Procesal-, el orden expositivo gira en torno al marco especifico del acceso a las fuentes de prueba digital en el orden jurisdiccional civil, en lo que tiene de contrapuesto al orden jurisdiccional penal, y en consecuencia algunos de los problemas que se exponen son comunes a todos los órdenes con excepción del penal, precisamente por razón de lo que tienen en común frente a aquel, aún dentro de las especificidades propias de cada uno.

Se estructura la exposición en tres apartados, analizándose en el primero de ellos la dificultad que representa para las partes procesales el acceso a las fuentes de prueba digital sin vulneración de derechos fundamentales, dada la inexistencia de una fase investigadora. Para ello, se adentra la ponente en el entorno específico de la prueba digital en relación con los poderes del Juez y de las partes; analiza el alcance de las exclusiones probatorias fuera del proceso penal y en concreto en el proceso civil; y se plantea por último la posible repercusión de la doctrina Falciani en su aplicación a la obtención de fuentes de prueba por particulares fuera de la órbita del orden penal.

En el segundo apartado, sugiere la autora otro enfoque del juego de poderes del Juez y de las partes en acceso a las fuentes de prueba, a propósito de dos recientes dictámenes de la Comisión Ética Judicial del Consejo General del Poder Judicial, que dan respuesta precisamente a la cuestión planteada en sendas consultas, que no es otra sino qué pasa con el uso de Internet por parte del Juez para buscar información sobre las partes, sus abogados o el objeto de la controversia en el proceso no penal.

Y por último, se cuestiona la autora si estamos avanzando hacia el modelo del Discovery, y en concreto si avanzamos hacia el modelo del Electronic-Discovery (eDiscovery) del Common Law, y si ello es deseable si la respuesta es sí, o si deberíamos avanzar en esa dirección si la respuesta es que no estamos avanzando en esa dirección. $\mathrm{Al}$ efecto, y tras la exposición breve del concepto de tales instituciones, enuncia la autora diversas razones que dan a entender que sí deberíamos avanzar en esa dirección, que pueden sintetizarse en la inoperancia de los instrumentos "paralelos" en nuestro sistema jurídico. Seguidamente procede a valorar los factores que refuerzan actualmente el acceso por las partes a las fuentes de prueba en nuestro ordenamiento jurídico, entre ellos la superación del Proceso civil adversarial a ultranza, la política legislativa de la Unión Europea y por último la incidencia de la globalización en la petición de Discovery a los Tribunales de EEUU desde países del Civil Law (con la mención específica de un reciente asunto planteado por ciudadanos españoles).

Concluye, finalmente, la Ponencia con la exposición de algunas Conclusiones. 
Ponencia: DILIGENCIAS INTRUSIVAS Y PRUEBA ILÍCITA, CON ESPECIAL REFERENCIA A LAS FUENTES DE PRUEBA DIGITAL EN EL ORDENAMIENTO CHILENO

Ponente: D. Raúl Núñez Ojeda, Profesor de Derecho Procesal de la Pontificia Universidad Católica de Valparaíso

En primer lugar, es preciso presentar el panorama actual de las diligencias intrusivas (o limitativas de derechos fundamentales) y la institución de la prueba ilícita en el campo del Derecho Procesal Penal. El análisis, además, se centra especialmente en los problemas que presenta la evidencia digital. Por lo señalado con anterioridad, se aborda la institución de los "hallazgos casuales" en las diligencias investigativas de incautación e interceptación de las comunicaciones digitales en Chile.

Así las cosas, luego de efectuar un breve análisis del principio de proporcionalidad y su relevancia en el tratamiento argumentativo de las diligencias intrusivas, se procede a abordar los hallazgos casuales desde una triple consideración: en primer término, realizando un análisis conceptual y metodológico de los hallazgos casuales en consideración a sus principales manifestaciones en el Derecho comparado, particularmente en Alemania, Italia y España; en segundo término, examinando los hallazgos casuales en Chile y sus manifestaciones jurisprudenciales más significativas, a partir de los artículos 215 (referido a la diligencia de entrada y registro) y 223 (relativo a la interceptación de comunicaciones telefónicas) del Código Procesal Penal; y, por último, abordando los hallazgos casuales de las comunicaciones digitales en Chile, sus problemas prácticos más relevantes, así como sus posibles formas de solución.

Comunicación: LA IDENTIFICACIÓN GENÉTICA Y FENOTÍPICA DEL AUTOR DEL DELITO. SU VALORACIÓN EN EL PROCESO PENAL

Comunicante: Da . Susana Álvarez de Neyra Kappler, Profesora Contratada Doctora de Derecho Procesal de la Universidad Autónoma de Madrid

La determinación y el aseguramiento del autor de la comisión de un hecho delictivo siempre ha sido un objetivo prioritario del proceso penal, para de este modo poder perseguir dicha conducta y satisfacer así el interés social en el castigo del infractor. Por ello ha sido una constante la preocupación de la identificación del delincuente, avanzándose en las técnicas destinadas a lograr dicho objetivo, más allá de la no siempre fiable -ni existente- prueba testifical, que se había alzado como la Regina probatorum. Y es que cada vez se confía más en los avances de la ciencia, que ayudan a hallar la verdad formalizada de los hechos.

Dicho objetivo no puede suponer en ningún caso que se les atribuya a las nuevas pericias un valor probatorio del que en ocasiones carecen, pues ello vulneraría, no sólo principios del proceso penal, sino verdaderos derechos procesales constitucionalizados. Es frecuente que el desconocimiento de las nuevas técnicas suponga darles un valor 
desmedido e inadecuado a sus resultados. Por tal motivo resulta fundamental que los operadores jurídicos tengan los conocimientos apropiados y suficientes para evitar incurrir en incorrectas valoraciones que chocarían con el debido proceso penal. No se puede pretender convertir a los peritos en jueces ni a los jueces en peritos, pero el conocimiento de quien va a imponer una pena que puede restringir el valor esencial de la libertad, debe ir acorde con los avances científicos y con el valor que se les da a sus resultados. Más allá de la prueba lofoscópica o, incluso, del uso del ADN en el proceso penal, se ha producido en los últimos tiempos una auténtica revolución en el ámbito de la genética forense, con el uso del fenotipado, que amenaza con dar a conocer, de una simple muestra genética, el origen biogeográfico de las personas, así como determinados rasgos físicos, que ayudarían a estrechar el círculo de los potenciales sospechosos de la comisión de un delito.

Comunicación: PRINCIPIO DE PROPORCIONALIDAD EN LA OBTENCIÓN, CESIÓN Y TRATAMIENTO DE DATOS PERSONALES EN MATERIA PENAL

Comunicante: $D^{\text {a }}$. María Elena Laro-González, Investigadora Contratada Predoctoral del Departamento de Derecho Procesal de la Universidad de Sevilla (FPI MINECO)

Desde hace algún tiempo las políticas europeas han puesto el foco de atención en la protección de los datos personales y en el intercambio de dichos datos en aras a la prevención, investigación, detección o enjuiciamiento de infracciones penales. Como consecuencia de ello, estas prioridades han quedado plasmadas en la Directiva (UE) 2016/680, de 27 de abril de 2016, del Parlamento Europeo y del Consejo, sobre protección de las personas físicas en lo que respecta al tratamiento de datos personales por parte de las autoridades competentes para fines de prevención, investigación, detección o enjuiciamiento de infracciones penales o de ejecución de sanciones penales, y sobre la libre circulación de dichos datos y por la que se deroga la Decisión Marco 2008/977/JAI del Consejo.

Las previsiones de la Directiva 2016/680/UE han venido precedidas de una importante jurisprudencia del TJUE, que ha sido decisiva en la elaboración de este texto normativo. De especial relevancia resultan las siguientes sentencias: la STJUE, de 8 de abril de 2014, en los asuntos acumulados C-293/12 y C-594/12, que tiene su origen en peticiones de cuestiones prejudiciales planteadas por Irlanda y Austria; la STJUE, de 21 de diciembre de 2016, en los asuntos acumulados C-203/15 y C-698/15, que tiene su origen en peticiones de cuestiones prejudiciales planteadas por Suecia y Reino Unido; y, la STJUE, de 2 de octubre de 2018, en el asunto C-207/16, que tiene su origen en las cuestión prejudicial planteada por la AP de Tarragona. En dichas sentencias se analiza la posible injerencia en los derechos de los investigados, conforme al principio de proporcionalidad.

Por su parte, el art. 4.1 de la Directiva 2016/680/UE enumera los principios rectores de la obtención, cesión y tratamiento de datos personales, lo cual viene a poner de manifiesto que la medida de investigación esté sujeta a los principios de especialidad, idoneidad, excepcionalidad, necesidad y proporcionalidad de la medida. No obstante, el art. 4.2 de la Directiva 2016/680/UE excepciona lo dispuesto en el art. 4.1, es decir, 
que se permite el tratamiento de los datos para el mismo fin pero en distinta causa penal. Esta excepción al principio de especialidad hay que ponerla en conexión con lo dispuesto en el art. 588 bis i) de la LECrim.

Por otra parte, debemos analizar el art. 588 ter j) de la LECrim, relativo a la incorporación de los datos electrónicos de tráfico o asociados, en cuanto al control de proporcionalidad atendiendo a la gravedad del delito. Del mismo modo, resulta necesario la valoración del requisito de la gravedad del delito en el análisis del art. 588 ter $\mathrm{m}$ ) de la LECrim, el cual regula la medida de investigación tendente a la identificación de los titulares de un número de teléfono o de cualquier otro medio de comunicación. Sobre ello, resulta relevante la interpretación de la Circular 2/2019, de la FGE, sobre interceptación de comunicaciones telefónicas y telemáticas.

Comunicación: LA EXPOSICIÓN DE LOS HIJOS MENORES EN REDES SOCIALES COMO PRUEBA PARA LA PRIVACIÓN DE LA PATRIA POTESTAD

Comunicante: $\mathbf{D}^{\mathbf{a}}$. Cristina Llamas Bao, Profesora Asociada de Derecho Administrativo de la Universidad de León. Abogada del ICAL

Cada vez es más frecuente, querer inmortalizar a través de fotografías y vídeos, los buenos momentos que hemos compartido con nuestros familiares y amigos, y hacer partícipes a los demás de ello. Pero el problema viene cuando, quienes figuran en esas fotografías y vídeos, son los hijos que son menores de edad.

La publicación en redes sociales de fotografías de los hijos y especialmente, menores de edad, ha pasado de ser el origen de una discusión familiar a utilizarse como principal argumento de las demandas en los procesos de familia, en particular, en aquellos en los que se discute lo relativo a la patria potestad.

Antes de contemplar con detalle esta problemática actual, se ha realizado un breve análisis jurisprudencial de los conceptos de titularidad de la patria potestad [SAP de Vizcaya (Sección 4a ) núm. 389/2019, de 6 de marzo, F.J 2º (JUR 2019\170806)]; ejercicio [ AAP de La Rioja (Sección $1^{\text {a }}$ ) núm. 150/2018, de 5 de diciembre (JUR 2019\37872)]; y privación de ésta [STS 14/2017, de 13 de enero (RJ 2017\15)].

Mención especial merece la SAP de Santa Cruz de Tenerife (Sección 1ª) núm. 356/2018, de 6 de julio (JUR2018\273535). Del estudio detallado de la misma se pueden extraer varias cuestiones: en primer lugar, que la publicación de las fotografías de una menos en redes sociales, implica una vulneración de su derecho a la propia imagen; en segundo lugar, que el consentimiento de los menores e incapaces deberá prestarse por ellos mismos si sus condiciones de madurez así lo permiten. Sin embargo, merece mención el hecho de que en esta sentencia no se termina de especificar qué se entiende por madurez, un problema que también encontramos en los procesos matrimoniales; en tercer lugar, que son los padres o tutores quienes respetarán los derechos al honor, a la intimidad y a la propia imagen de sus hijos; y en cuarto lugar, para este Tribunal la decisión de publicar o no la fotografía de la menor constituye un asunto que, aunque no 
sea de «urgente necesidas», sí es de «especial trascendencia» y que requiere, por tanto, del consentimiento de ambos progenitores.

Comunicación: EL VALOR PROBATORIO DE LA INFORMACIÓN OBTENIDA DE UN ORDENADOR DE USO COMPARTIDO: A PROPÓSITO DE LA STS 287/2017 Comunicante: $\mathbf{D}^{\mathrm{a}}$. María del Pilar Martín Ríos, Profesora Titular de Derecho Procesal de la Universidad de Sevilla

Se procede a analiza el valor probatorio que puede atribuirse, en el seno de un proceso penal, a la aportación de datos alojados en un dispositivo de almacenamiento masivo de información digital. Para ello, habremos de tener presente que el debido respeto al "derecho al propio entorno virtual o digital" - derecho fundamental de nueva generación, fruto de una construcción doctrinal y jurisprudencial- operará como límite a este género de actuaciones. Podremos, asimismo, trazar algún paralelismo entre este novedoso derecho y el "derecho al domicilio informático" italiano.

Con independencia de la importancia que pudiera revestir quién realizase tal aportación probatoria (fuera un particular -usuario o no de dicho dispositivo-, fuera un agente de la autoridad), la principal finalidad de nuestro estudio es examinar la concurrencia de una circunstancia peculiar: que los dispositivos de donde se extrajese la información que pretende usarse en el proceso fuesen de uso compartido. Para tal fin, examinaremos críticamente la STS 287/2017, de 19 de abril, lo que nos permitirá conocer -y objetar- cuál es la postura de nuestro Tribunal Supremo respecto de la protección de que es merecedora la información allí ubicada.

Comunicación: EXCLUSIONES PROBATORIAS A INSTANCIA DE LA PERSONA JURÍDICA

Comunicante: D. Felipe Pérez del Valle, Profesor Asociado de Derecho Procesal Penal de la Universidad de León. Abogado del ICAL

Los medios de investigación electrónica autorizados por la Ley Orgánica 13/2015, de 5 de octubre, de modificación de la Ley de Enjuiciamiento Criminal para el fortalecimiento de las garantías procesales y la regulación de las medidas de investigación tecnológicas, implican un altísimo nivel de agresión en la esfera de la persona sospechosa de haber cometido un delito. Así que, para evitar una posible dictadura tecnológica a manos de ciertos "poderes", se ha de limitar ese uso ilimitado de tales facultades de investigación mediante un "uso adecuado del Derecho", salvaguardando los derechos fundamentales y las libertades públicas, a través de las exclusiones probatorias de la investigación y prueba electrónica que vulnere cualquier derecho fundamental, teniendo en cuenta, también, del que empieza a denominarse "derecho al entorno virtual", tanto del ciudadano individual como del grupo o colectivo constituido válidamente, incluyendo a la persona jurídica, ya sea de pequeña o gran dimensión. 
Debido a ello, establecida la responsabilidad penal de la persona jurídica por la comisión, así como el reconocimiento de que goza de determinados derechos fundamentales, entre ellos algunos de los regulados en el artículo 18 de la Constitución, por tanto, al regir para ella los mismos principios penales que para la persona física, en el supuesto de estar sometida a algún medio de investigación limitativa de los derechos reconocidos en el art. $18 \mathrm{CE}$, podrá instar la exclusión de una prueba ilícitamente obtenida en base al art. 24. 2 CE y 11. 1 LOPJ.

Comunicación: PROCESO PENAL Y VOLATILIDAD/MUTABILIDAD DE LAS FUENTES DE PRUEBA ELECTRÓNICAS: SOBRE LA CONVENIENCIA Y EL MODO DE ASEGURARLAS EFICAZMENTE

Comunicante: $D^{a}$. Eva Isabel Sanjurjo Ríos, Profesora Contratada Doctora de Derecho Procesal de la Universidad de León

En un corto período de tiempo hemos pasado de una sociedad en la que todos realizábamos un muy escaso o limitado uso de la informática o de cualquier dispositivo electrónico, a un período en el que cada vez resulta más difícil "desengancharse" de la tecnología e informática. Y todavía más de Internet, fuente instantánea y abundantísima de información.

El mundo del Derecho no ha sido ajeno a esta realidad y, por ende, tampoco el Derecho Procesal Penal. En escaso tiempo, el proceso penal ha visto cómo las nuevas tecnologías de la información y comunicación han dejado su impronta en él de dos formas diferentes, a la par que no excluyentes entre sí: como medio para la comisión de hechos delictivos (la llamada "ciberdelincuencia"); y como instrumento esencial para la investigación del delito y su autoría, sin obviar su utilidad como prueba de la acusación o de la defensa.

Como resultado de lo anterior, resulta harto frecuente que durante la sustanciación de la fase de instrucción de un proceso penal surja la necesidad de acceder a una determinada información electrónica, independientemente del soporte en el que se halle. Y más aún, la premura de su custodia procesal, en cuantoa que las fuentes de prueba digital se caracterizan, precisamente, por la altísima rapidez con la que pueden desaparecer de la red o del soporte que las almacenan (volatilidad) y su sencilla modificación por parte de quienes tienen unos conocimientos mínimos en materia informática o tecnológica (mutabilidad).

La constatación de esa volatilidad y mutabilidad de las fuentes probatorias digitales justifica esta breve investigación, a fin de poder profundizar sobre las medidas procesales adecuadas para garantizar la autenticidad e integridad de aquéllas y evitar que la alta volatilidad y la sencilla mutabilidad se convierta en un verdadero escollo durante el enjuiciamiento de cualquier conducta delictiva. Singularmente, imposibilitando que dicha fuente sea aportada y propuesta como prueba durante la fase del juicio oral. 
Finalmente, la comunicación presentada se estructura en torno a dos supuestos distintos, en función de si la fuente de prueba digital es introducida por alguna de las partes durante la instrucción o, en cambio, es resultado de la actividad investigadora de la autoridad judicial. Para el examen de cada uno de ellos, y en la medida de lo posible, se parte de la regulación legal existente al efecto en la decimonónica LECrim, resultando oportuno también reparar en algunas de las importantes y loables -no todas - reformas procesales que se introdujeron en el 2015 sobre dicha Ley.

Comunicación: LA CADENA DE CUSTODIA EN LA PRUEBA DIGITAL: ESPAÑA VS. EEUU

Comunicante: $\mathrm{D}^{\mathrm{a}}$ Margarita Simarro Pedreira, Doctoranda del Departamento de Derecho Procesal de la Universidad de León

La correcta conservación de la cadena de custodia cuando se trata de las evidencias digitales es una cuestión que ha planteado muchos problemas en la práctica debido a la gran cantidad de formas de comunicación existentes en la actualidad, la volatidad de los datos, la dificultad para identificar la veracidad de los mismos y los avances de la tecnología.

La cantidad de datos que circulan por el ciberespacio puede afectar a los derechos reconocidos en el art. 18 de nuestra Constitución, así el derecho al secreto de las comunicaciones, el derecho a la intimidad o el derecho a la protección de datos de carácter personal, pero también afectan a otros nuevos derechos acuñados por la jurisprudencia como son el derecho al entorno digital o derecho al olvido digital que deberían tener acogida de forma independiente en nuestra Constitución.

La cadena de custodia se torna fundamental para que la prueba digital llegue al proceso con las máximas garantías de autenticidad, de ahí que sea imprescindible que se cumplan unos protocolos estrictos de manipulación, conservación y custodia. Sin embargo, en nuestro país existe una falta de regulación específica que determine la metodología a seguir en cuanto a su aseguramiento. Si bien existen algunas normas de carácter administrativo aisladas y algunos artículos de la LECrim que se refieren a la prueba en la investigación tecnológica no contemplan aspectos específicos de cómo realmente se tiene que llevar a cabo esa recogida y conservación de vestigios digitales.

En España, y hasta que no se promulgue una legislación específica al respecto, hay que remitirse a la doctrina para poder encontrar un proceso escrupuloso en materia de cadena de custodia y, en especial, de la que se debe de aplicar a la prueba digital.

En EEUU la situación es muy diferente, a pesar de tratarse de un Estado Federal en el que coexisten dos sistemas judiciales diferentes (el Federal aplicable a toda la nación y el Estatal en el que cada Estado tiene su propia legislación), sí existe una norma federal, La Federal Rules of Evidence, y varias guías elaboradas por el Departamento de Justicia de los Estados Unidos que son utilizadas tanto por la policía como por las diversas Agencias Federales a la hora de recabar muestras que se refieren específicamente a la cadena de custodia de la evidencia digital. 